\title{
Multi-wavelength Flashes from GRBs
}

\author{
Dieter H. Hartmann \\ Department of Physics and Astronomy, Clemson University, Clemson, SC 29634, USA
}

\section{Debates}

The mystery of the nature and origin of gamma-ray bursts (GRBs) has persisted over two decades of intensive research, observational and theoretical. The basic problem is the absence of quiescent or transient counterparts at other wavelengths, resulting in distance scale uncertainties of more than 10 orders of magnitude. Searches for counterparts are hampered by the lack of sufficiently accurate localizations and by a lack of criteria that uniquely identify the expected appearance of counterparts (although see Meszaros \& Rees 1993, Meszaros et al. 1994). The detection of archival optical transients (OT's) inside $\gamma$-ray burst error boxes suggested burst recurrence and generated hope for a breakthrough in the $\gamma$-ray burst counterpart search (e.g., Schaefer 1990). However, counterparts of these OT's have never been identified, and the reality of several archival images was questioned (Zytkow 1990, but see Schaefer 1990). The search for multi-wavelengths transients has recently been extended to neutrino- and gravitational wave emission. The status of multiwavelength surveys for quiescent or transient counterparts prior to 1988 was reviewed by Hartmann \& Woosley (1988). Here we update that review and discuss "optical" emission in view of the current debate on the burster distance scale. Other recent reviews are by Schaefer (1994) and Greiner (1995).

The recent transition from the original paradigm of Galactic neutron stars to the new paradigm of cosmological sources was generated by global statistical properties of GRBs. The non-uniform brightness distribution combined with the angular isotropy argues against Galactic source distributions and favors cosmological origins. BATSE data improve the global statistical constraints at an approximate rate of one burst per day, but the detection and unambiguous identification of even a single GRB counterpart would carry more weight than all statistical constraints taken together. If GRBs are indeed associated with galaxies, as some models suggest, we would expect to find and identify them inside the error boxes, at least for very accurate positions. We take inventory of GRB locations and discuss implications for the models. 
From their statistical properties we conclude that bursts either reside in an extended halo $(100 \mathrm{kpc})$, or are extragalactic $(100 \mathrm{Mpc})$ or possibly cosmological. Very local origins (Oort cloud, nearby section of Galactic spiral arms, etc.) appear to be ruled out by the data (e.g., Briggs et al. 1995). The multiwavelength signatures of GRBs obviously depend on the nature of the energy release mechanisms and the environment of the source if reprocessing of some fraction of the radiation should occur. The archival data on optical transients suggest, if one believes their reality, that a significant fraction of the burst energy is converted to low energy bands. These data also suggest that bursts do repeat on timescale of order years. Is that claim consistent with the observed angular distribution? There are only three well established cases of burst repetition: GB790305b (the "March 5 event"), GB790107 (the "soft repeater"), and GB790324. All three events have in common that they are relatively short in duration and that their mean photon energies are less than average. These characteristics led to a proposed class of "soft gamma-ray repeaters (SGRs)", that is different from the class of "classical bursts (GRBs)" (Kouveliotou 1994). Much progress has recently been made in the identification of their Galactic counterparts, but space limitations of this paper prevent a more detailed discussion of this sub-group.

There is no convincing evidence for recurrence of classical GRBs (Meegan et al. 1995), but the analysis is somewhat hampered by poor angular resolution. Model-dependent statistical estimates of recurrence from pre-BATSE data suggested time scales of $\sim 10 \mathrm{yrs}$, but analyses of early BATSE data led to the suggestion of much shorter recurrence times. If bursts indeed originate at large redshifts, the merger of compact stellar objects (neutron stars, black holes, white dwarfs) appears to be a natural GRB site. Since these events must occur in nature and since they are rare, we must sample a significant fraction of the universe to be consistent with the observed event rate of $\sim 10^{3}$ per year. Mergers are expected to occur at a rate of $\sim 10^{-6} \mathrm{yr}^{-1}$ per host galaxy, so that recurrence over the lifetime of $\gamma$-ray detectors is not expected. Positional coincidences on the sky are thus expected to be random. Deviations from randomness can be caused by non-uniform sampling of the sky, spatial clustering of the underlying source population, large scale anisotropies, or burst repetition. Since the latter case would rule out some of the currently favored cosmological scenarios, the question of burst recurrence needs careful consideration.

Quashnock \& Lamb (1993) presented arguments for clustering on small angular scales for BATSE burst data contained in the $1 \mathrm{~B}$ catalog and suggested that multiple recurrences of a significant fraction of bursts were responsible for the observed excess. A possible correlation of bursts in both space and time was suggested by Wang \& Lingenfelter (1993) who isolated GBS $0855-00$ as a potential location of multiple recurrences. If a significant fraction of bursts indeed repeat on short timescales, the angular distribution would be distorted on scales comparable to the angular resolution of the instrument. Localization accuracy of $B A T S E$ is currently limited by systematic effects to $\sim 4^{\circ}$. Low order multipole analysis does not resolve these scales, so that different methods must be employed to test for small angle clustering. Two tools suitable for this task 
are the angular correlation function and the nearest neighbor statistic. Using these methods, and others, the BATSE 2B data were searched for recurrences (Meegan et al. 1995, Brainerd et al. 1995, Hartmann et al. 1995). Although recurrence of some bursts can not be ruled out, these studies suggest that frequent recurrence of a large fraction of all bursts does not occur.

\section{Localizations}

The largest and most uniform database of burst positions is now available from the BATSE experiment. Over 1,200 burst have been recorded since the launch of Compton Observatory (CGRO). BATSE determines directions to bursts from the relative countrates in the eight detector modules, using an essentially $\cos (\theta)$ law for the dependence of count rate on the angle of incidence. Scattering of burst photons in the spacecraft as well as reflection off the Earth atmosphere create a systematic uncertainty that amounts to about $4^{\circ}$. The statistical uncertainties can be less than a degree for very bright bursts, but both sources add in quadrature! Independent positions come from triangulations with the Inter Planetary Network (IPN), solar flares, and a few independently measured burst locations from COMPTEL and EGRET. In general, the BATSE positions alone are not accurate enough to allow deep ground-based observations. While positions with degree resolution may soon be attainable for some bursts, the majority of bursts is too faint to yield such position accuracies. However, we do not yet seek counterparts for all bursts - we are interested in finding convincing candidates for at least a few bursts.

While the positions from $C G R O$ are useful in many ways, the search for counterparts requires a location accuracy that must be orders of magnitude better. There are two separate approaches to reducing the sizes of error boxes. The first method uses $\gamma$-ray data directly, and the second method attempts to obtain accurate locations through a wide field of view search for simultaneous flares in the optical band. Better positions from $\gamma$-ray data are obtained through the classical method of burst timing with a network of widely separated spacecraft (Hurley, Cline, and collaborateurs pioneered this approach). The accuracy of triangulation depends on burst brightness and structure of the lightcurves, and also on the number and sensitivity of available detectors. Despite substantial variations typical burst locations from the IPN are of order arcminutes (e.g., Hurley et al. 1993). The shapes of the error boxes also vary. Burst locations may be improved when IPN/BATSE positions are combined with single detector localizations from COMPTEL, EGRET, WATCH, or SIGMA. The next generation IPN could accomplish arcsecond resolution if detectors on a future Pluto flyby mission could be equipped with GRB detectors (Hurley \& Cline 1994).

The other approach is based on a rapid, though coarse, localization of a burst combined with rapid ground-based searches for optical flares. The combination of BATSE and COMPTEL allows, although infrequently due to the limited FoV of COMPTEL, follow-up studies within hours of a burst (Kippen et al. 1994). A worldwide web of optical and radio observatories, coordinated from NMSU 
(Harrison et al. 1995), is alerted by the BATSE/COMPTEL system and immediately surveys the COMPTEL field with wide FoV telescopes. The monitoring of the burst field continues for a considerable period after the burst. Detection of optical or radio transients would automatically lead to astrometric positions, which could then be studied further.

Another system with similar concepts is the BATSE-BACODINE-GROCSE alliance (Barthelmy et al. 1994). This system allows a rough $\left(10^{\circ}\right)$ localization of a GRB while it is bright, i.e., on a timescale of seconds. This information is used to point a small, but rapidly moving telescope with wide field of view. The prototype GROCSE system is located at LLNL in California. Detection of optical flares would yield the desired burst locations. If the BACODINE/GROCSE system (and its relatives) are not successful, hope for better positions rests on the shoulders of the HETE experiment (Ricker et al. 1988), which will be launched in late 1995. This experiment provides simultaneous multiwavelengths coverage in the $\gamma$-ray, $\mathrm{X}$-ray, and UV bands. The detection of X-rays will yield arcminute positions, and if UV emission is detected the accuracy will be of order arcseconds! For various cosmological fireball models Meszaros et al. (1994) estimated the IR, optical, UV, X-ray, and $\gamma$-ray fluences. Their results suggest that HETE would detect low-energy emission from cosmological GRBs under a wide range of circumstances. In the future, new concepts such as the ETA (Ricker 1990), an array of detectors in solar orbit, could achieve arcsec resolution directly from $\gamma$-ray lightcurves. The ETA experiment would obtain precise GRB positions for $\sim 10^{3}$ GRBs $y^{-1}$. Eventually accurate enough positions must contain uniquely identifiable counterparts, otherwise new physics is required. There could be a time in the near future when routine studies of GRB counterparts for thousands of sub-arcsec positions are used to reveal aspects of the universe that we have so far only glimpsed.

\section{Quiescent counterparts}

This field was reviewed at the 2nd Huntsville workshop by Schaefer (1994). The survey of GRB error boxes in the X-ray band was continued with ROSAT (Boer et al. 1993; Greiner et al. 1995). Although a few sources in or near GRB error boxes were found, no clear correlation is apparent.

Longstanding programs to survey and monitor GRB locations in the optical are being carried out at various observatories. The USNO (Flagstaff Station) survey of older IPN positions was completed (Vrba et al. 1995). While the final census of objects in this survey was consistent with the expected numbers of normal stars and galaxies several potentially interesting objects were found. In several cases moderate evidence for a GRB-QSO connection was found (Vrba et al. 1994). That such an association can not hold for all GRBs has been argued by Schaefer (1994) and Fenimore et al. (1993), based on the absence of bright host galaxies in most error boxes. The observed magnitude limits typically imply source distances in excess of $\sim 1 \mathrm{Gpc}$, but a realistic luminosity function has yet to be used to derive more rigorous distance limits. Results from an optical survey 
program at New Mexico State's Blue Mesa Observatory were presented in recent papers (Harrison et al. 1994a,b). Again, no obvious counterparts were identified. One new development occured in the case of FY Aql, which is a Mira variable star (in the field of GRB790331) surrounded by a reflection nebula (Hartmann \& Pogge 1987, Schaefer 1990). Recent observations (Irwin \& Zytkow 1995) find no trace of the nebula and obtain evidence for binary nature of this star. These data may turn FY Aql again into an interesting counterpart candidate.

Searches for quiescent $\gamma$-ray burst counterparts in the extreme UV were carried out with the ROSAT XUV camera (Owens et al. 1993) and the EUVE experiment (Hurley et al. 1995). The former study surveyed the regions of 12 GRBs and 9 OTs, without evidence for emission above $10^{-11} \mathrm{ergs}^{\mathrm{cm}} \mathrm{Cm}^{-2} \mathrm{~s}^{-1}$. In the latter study the field of GRB920325 was studied 17 months after the burst, and again no steady source was found inside or near the burst error box. The upper flux limit derived from this 40ks observation was $310^{-14} \mathrm{ergs} \mathrm{cm}^{-2} \mathrm{~s}^{-1}$ in the 40-190 $\AA$ band. The natural source candidates we may expect to identify in the EUV are nearby compact objects with or without accretion disks around them. Detection would depend on the state of the accretion disk and the surface temperature of the star. The non-detection leads to highly model dependent constraints, but it is perhaps save to say that no normal neutron star with $\mathbf{T} \sim$ $10^{5-6} \mathrm{~K}$ within a few $100 \mathrm{pc}$ of the Sun would have gone undetected. Similar distance limits follow from the X-ray constraints discussed above.

\section{Transient counterparts}

As discussed above, accurate burst positions are now becoming available on much shorter timescales, which opens the possibility of rapid or even very rapid multiwavelengths searches for transient counterparts. Most of these programs require international collaboration and the coverage in wavelength, time, and flux relative to the burst varies dramatically from burst to burst. Within the limits of this review I select a few highlights of this very active field.

VHE: The $E^{2} F_{E}$ spectrum of GRBs often peaks in the $\sim 1 \mathrm{MeV}$ regime, but significant "tails" can extend to $\sim 100 \mathrm{MeV}$ (demonstrated with $S M M$ ), and in some cases into the $\mathrm{GeV}$ range (recent $E G R E T$ data). This high energy emission provides severe constraints on cosmological redshifts to GRB sources because at these energies $\gamma$-rays efficiently pair-create through interaction with the CMB radiation field and the intergalactic IR background produced by galactic starlight. The EGRET observations of GRB940217 (Hurley et al. 1994) implies that in some cases apparently unattenuated spectra extend to $\sim 20 \mathrm{GeV}$. This observation emphasizes the need for ground-based searches at even higher energies. Detection in the $\mathrm{TeV}$ range by means of atmospheric Cerenkov detectors would severely constrain distances of cosmological scenarios. Searches have been carried out at the Whipple Observatory (Chantell et al. 1993, Connaughton et al. 1994 ) in the TeV band, and at the Cygnus-I air shower aray (Alexandreas et al. 1994, Schnee et al. 1995) above $100 \mathrm{TeV}$. No detection has yet been claimed. Prospects for a breakthrough in this band are very good for the MILAGRO 
Gamma Ray Observatory currently under construction at LANL. This waterCerenkov detector operates above $\sim 250 \mathrm{GeV}$ and should be the first detector in this band sensitive enough to detect all GRBs with unattenuated spectra with roughly $\mathrm{E}^{-2}$ extrapolation of CGRO data (Shoup et al. 1995).

Optical: One of the first bursts with rapid localization was GRB920501, but no unique counterpart was found among the various objects detectable in optical and X-ray observations (Hurley et al. 1994). In the case of GRB930131 an international campaign searched for optical and radio emission only 7 hours after the event (Schaefer et al. 1994). Again, no obvious counterparts were found. An even faster survey was possible for GRB940301, which was observed in the radio band one hour after the burst and in the optical band seven hours after the burst (Harrison et al. 1995). These results from the NMSU Rapid Response Network are impressive, but still did not detect transient emission that one could relate to the GRB. Clearly, simultaneous observations are the final frontier. OT searches on archival plates are a useful, but often unreliable source of information (see Hudec 1993 for a recent review), and usually the images of OTs are well removed in time from the GRB event. Greiner et al. (1994) has compiled a list of bursts for which simultaneous data are available. The absence of optical flares in these cases places an upper limit on the ratio of optical to $\gamma$-ray flux, $R_{0, \gamma} \leq$ 0.5. Current efforts to further tighten these constraints are underway at LLNL, where the GROCSE system can respond to a BACODINE location in a few seconds (under favorable circumstances). Several bursts have now been observed with this system (but only with partial coverage of the error box) down to a magnitude limit of $\sim 7$. No optical images were found, yielding similar limits to those discussed by Greiner. Results from the ETC Observatory were discussed at this symposium by Vanderspek and by Greiner (1995).

Radio: If only a small fraction of the burst energy emerges in the radio band, the dispersion in the ISM of our Galaxy and the intergalactic medium (IGM) could result in arrival time delays of $\sim 1$ hour at radio frequencies $\nu \sim 25 \mathrm{MHz}$. If accurate locations can be derived quickly, which is now possible with the $B A$ $C O D I N E$ response network, there could be enough time to point radio telescopes to the burst direction. Radio detection at two frequencies would then yield the dispersion measure (DM) and thus provide an estimate of the burster distance (Palmer 1993), just as in the case of radio pulsars in our Galaxy. Paczynski \& Rhoads (1993) considered the interaction of relativistically expanding fireballs with interstellar or intergalactic matter. Due to collisions between matter in the GRB wind and in the surrounding medium a fraction of the ordered kinetic energy will be randomized and some part will eventually be radiated away in the form of low energy photons. At late stages in the development of such "radio fireballs" synchrotron emission dominates over inverse Compton cooling of the electrons and one can estimate the radio spectrum assuming that the emission is incoherent. If reheating of the expanding shell generated an electron distribution function that is a power law in electron energy, $n(E) \propto E^{-p}$, synchrotron emission from optically thin regions produces a power law spectral intensity with slope $\alpha=-(p-1) / 2$. Self absorption in optically thick regions changes this 
slope to $\alpha=5 / 2$, independent of the electron distribution function and different from the thermal Rayleigh-Jeans limit $\alpha_{\mathrm{RJ}}=2$. Paczynski \& Rhoads carry out the transformation from the restframe of the emitting shell and find that GRBs could generate radio fluxes above $0.1 \mathrm{Jy}$ at frequencies of a few $\mathrm{GHz}$. If burst emission is strongly beamed, however, the reduced energy requirements (usually a blessing) could render the radio transients undetectable. Assuming isotropic emission, the peak of the radio emission would occur with a time delay, $\Delta t$, relative to the beginning of the fireball

$$
\Delta t \sim 0.37 E_{53}^{1 / 2} \varrho_{-24}^{1 / 2} \nu_{\mathrm{GHz}}^{-3 / 2} \text { yrs. }
$$

While radio bursts from GRBs at cosmological distances are likely to show substantial delays, bursts in the Galactic halo require a much smaller primary energy and might thus be accompanied by simultaneous radio bursts. A radio signal at frequency $\nu$ propagates through a cold electron gas (number density $n$ ) with group velocity

$$
\beta=\frac{v}{c}=\left[1-\left(\frac{\nu_{p}}{\nu}\right)^{2}\right]^{1 / 2},
$$

where $c$ is the speed of light in vacuum, and $\nu_{p}$ the plasma frequency. The time delay, relative to a wave propagating with the speed of light, is

$$
\delta \mathrm{t}(\nu)=\int_{0}^{l} d x(c-v) c^{-2}=\frac{1}{c} \int_{0}^{l} d x(1-\beta),
$$

In the limit $\nu \gg \nu_{p}$ the time lag relates to the dispersion measure as

$$
\delta \mathrm{t}(\nu)=\frac{\mathrm{e}^{2}}{2 \pi \mathrm{m}_{\mathrm{e}} \mathrm{c} \nu^{2}} \int_{0}^{l} d x \mathrm{n} \sim \frac{\mathrm{DM}}{240 \nu_{\mathrm{GHz}}^{2}} .
$$

where the dispersion measure ( $\mathrm{DM}=$ total electron column density) is commonly measured in the units $\mathrm{pc} \mathrm{cm}^{-3}$. The Galactic free electron distribution gives typically $\mathrm{DM} \sim 10^{2} \mathrm{pc} \mathrm{cm}^{-3}$ in the Galactic plane, but can reach $\sim 10^{3} \mathrm{pc}$ $\mathrm{cm}^{-3}$ for directions close to the Galactic center. If the radio source is located at cosmological distances, the additional electrons in the intergalactic medium (IGM) add to the delay. Because the plasma frequency is density dependent, the simple $\delta t-\mathrm{DM}$ relationship does no longer hold, but it is straightforward to derive a very similar form

$$
\delta t(z)=\frac{\mathrm{DM}_{0}}{240 \nu_{\mathrm{GHz}}^{2}}\left(\frac{2}{\Omega}\right)
$$

where $\mathrm{DM}_{0}=2.4610^{4} \mathrm{~h}_{75} \Omega_{b}$ is the dispersion measure scale for a Friedman universe with baryon fraction $\Omega_{b}$. A radio transient emitted at $z \sim 1$ thus experiences a delay, relative to the $\gamma$-ray bursts, of over one minute. If the GRB source region itself is enshrouded by an electron cloud of significant column density, the delay would increase. Of course, radio emission could also be delayed due to intrinsic evolution of the radiating source region. A recent search for delayed radio emission was carried out for GRB940301 (Frail et al. 1994). Wide field radio 
images at $0.4 \mathrm{GHz}$ and $1.4 \mathrm{GHz}$ were obtained at the DRAO Synthesis Telescope between $3-15$ and 26,47 , and 99 days after the burst. No flaring object brighter than $3.5 \mathrm{mJy}$ was seen, suggesting that some model parameters were chosen too optimistically.

Gravitational waves (GW): If neutron star mergers cause GRBs, there should be a coincident burst of gravitational radiation. The brightest bursts could be detectable with $L I G O$, expected to be operational in 1999. The first phase LIGO may not be sensitive enough to detect such GRBs past $\sim 10 \mathrm{Mpc}$, but LIGO upgrades will increase its sampling distance with time. The knowledge of GRB arrival times reduces detector backgrounds and thus improves sensitivity. Kochanek \& Piran (1993) estimate that failure to observe GRB-GW pairs with a rate of $1 \mathrm{yr}^{-1}$ above a strain of $\sim 10^{-22}$ would rule out the merger hypothesis.

Neutrino bursts: The merger of two neutron stars releases $\sim 10^{53}$ ergs of energy, most of which is radiated away by thermal neutrinos of all flavors, and a small fraction of this energy may be converted to power the GRB. Neutrino energies are $\sim 10 \mathrm{MeV}$, and thus hard to detect. Much higher energies (GeV) could result when fireballs interact with matter, causing production of pions that decay, amongst other particles, to neutrinos (Paczynski \& Xu 1994). Neutrino bursts from superconducting strings at large redshifts were discussed by Plaga (1994), but the EGRET observations of $\mathrm{GeV}$ photons argue against redshifts in excess of 10-100. Searches for simultaneous neutrino bursts were carried out by the Soudan collaboration (DeMuth et al. 1994), the $I M B$ collaboration (LoSecco 1994), and at Mont Blanc (Aglietta et al. 1993). No correlation was found to a fluence level of $\sim 310^{4}$ ergs $\mathrm{cm}^{-2}$.

\section{References}

Alexandreas D.E., 1994, ApJ 426, L1

Anglietta M. et al., 1993, 23rd ICRC, 1, 69

Barthelmy S.D., 1994, in Gamma-Ray Bursts, G.J. Fishman, K. Hurley, \& J. Brainerd (eds.), AIP 307, 643

Boer M. et al., 1993, A\&A 277, 503

Brainerd J.J. et al., 1995, ApJ (in press)

Briggs M. et al., 1995, ApJ (in press)

Chantell M. et al., 1993, in Compton Gamma Ray Observatory, AIP 280, 833

Connaughton V. et al., 1994, in Gamma Ray Bursts, G.J. Fishman, K. Hurley, \& J. Brainerd (eds.), AIP 307, 470

DeMuth, et al., 1994, in Gamma Ray Bursts, AIP 307, 475

Fenimore E.E. et al., 1993, Nature 366, 40

Frail, D.A. et al., 1995, ApJ 437, L43

Greiner J., 1995, Proc. 17th Texas Symp. on Rel. Astrophysics (in press)

Greiner J. et al., 1994, in Gamma Ray Bursts, G.J. Fishman, J.J. Brainerd \& K. Hurley (eds.), AIP 307, 408

Greiner J., Boer M., Kahabka P., Motch C., Voges W., 1995, in The Lives of Neutron Stars, A. Alpar (ed.), NATO ASI 450, Kluwer, Dordrecht, p. 519 
Harrison T., McNamara B., Klemola A., 1994a, AJ 107, 254

Harrison T., McNamara B., Klemola A., 1994b, AJ 108, 600

Harrison T. et al., 1995, A\&A (in press)

Hartmann, D.H., Pogge, R., 1987, ApJ 318, 363

Hartmann, D.H., Woosley, S.E., 1988, in Multiwavelengths Astrophysics, F. Córdova (ed.), Cambridge Univ. Press, Cambridge, p. 189

Hartmann, D.H. et al., 1995, ApJ (in press)

Hudec R., 1993, A\&AS 97, 49

Hurley K. et al., 1993, A\&AS 97, 39

Hurley K. et al., 1994, ApJS 92, 655

Hurley K. et al., 1995, ApJ (in press)

Hurley K., Cline, T., 1994, in Gamma Ray Bursts, G.J. Fishman, K. Hurley, \& J. Brainerd (eds.), AIP 307, 653

Hurley K. et al., 1994, Nature 372, 652

Irwin M., Zytkow A.N., 1995, ApJ (in press)

Kippen M. et al., 1994, in Gamma Ray Bursts, eds. G.J. Fishman, K. Hurley, \& J. Brainerd (eds.), AIP 307, 418

Kochanek, C.S., Piran, T., 1993, ApJ 417, L17

Kouveliotou C., 1994, Nature 370, 26

LoSecco, J.M., 1994, ApJ 425, 217

Meegan C.A. et al., 1995, ApJ (in press)

Meszaros P., Rees, M.J., 1993, ApJ 418, L59

Meszaros P., Rees, M.J., Papathanassiou, H., 1994, ApJ 432, 181

Owens A. et al., 1993, in COMPTON Observatory, M. Friedlander, N. Gehrels \& D.J. Macomb (eds.), AIP 280, 798

Palmer D.M., 1993, ApJ 417, L25.

Plaga, R., 1994, ApJ 424, L9

Paczynski P., Xu G., 1994, ApJ 427, 708

Paczynski P., Rhoads J., 1993, ApJ 418, L5

Quashnock J.M., Lamb D.Q., 1993, MNRAS 265, L59

Ricker G. et al., 1988, in Nuclear Spectroscopy, N. Gehrels, \& G.H. Share (eds.), AIP 170,407

Ricker G., 1990, in High Energy Astrophysics, P. Joss (ed.), AIP 211, 365

Schaefer B.E., 1994, in Gamma Ray Bursts, G.J. Fishman, K. Hurley, \& J. Brainerd (eds.), AIP 307, 382

Schaefer B., 1990, ApJ 364, 590

Schaefer B. et al., 1994, ApJ 422, L71

Schnee, R., et al. 1994, in Gamma Ray Bursts, G.J. Fishman, K. Hurley, \& J. Brainerd (eds.), AIP 307, 481; see also BAAS 26, 1508

Shoup A. et al., 1994, BAAS 26, 1335

Vrba F.J. et al., 1994, ApJ 424, 68

Vrba F.J., Hartmann D.H., Jennings M., 1995, ApJ (in press)

Wang V., Lingenfelter R.E., 1993, ApJ 416, L13

Zytkow A., 1990, ApJ 359, 138 